ISSN electrónico: 2445-1355

DOI: http://dx.doi.org/10.14201/fj2020514754

\title{
ESTUDIO DE REVISIÓN DE LAS VENTAJAS \\ E INCONVENIENTES DE LOS SISTEMAS \\ PERSONALIZADOS DE DOSIFICACIÓN (SPD) Y \\ EVALUACIÓN DE SU ADECUACIÓN AL PERFIL DEL PACIENTE Y TIPO DE FARMACIA
}

\section{Study Review of the advantages and disadvantages of Dose Administration Aids (DDA) and evaluation of their adequacy to the patient's profile and type of pharmacy}

Ana Belén MAESTRE HERNÁNDEZ; María GONZÁLEZ VALDIVIESO²; Josep ARASA³; Elsa LÓPEZ PINTOR ${ }^{4}$

${ }^{1}$ Universidad Miguel Hernández de Elche; anabelenmae@gmail.com; 657970 598;

2 maria@farmacialabarbera.com; 616635 044; ${ }^{3}$ info@dosificacion.es; 670548438

4 elsa.lopez@goumh.umh.es; 676463209

RESUMEN: Los Sistemas Personalizados de Dosificación (SPD) son una herramienta útil para mejorar la adherencia terapéutica desde la farmacia comunitaria. Sin embargo, existen en el mercado multitud de sistemas con características diferenciadoras que es necesario conocer para poder seleccionar la que mejor se adapte al servicio de SPD.

El objetivo de este trabajo ha consistido en realizar una revisión de los diferentes tipos de SPD que existen, analizar las ventajas e inconvenientes de cada uno y evaluar su adecuación en función del uso deseado, perfil de paciente y tipo de farmacia. Para dar respuesta a estos objetivos, se ha realizado una revisión de la información por diferentes fuentes y métodos. Se han identificado tres tipos de SPD: manuales, semiautomáticos y automáticos, diferenciándose fundamentalmente en el cierre del blíster, el tipo de sellado, la duración, el personal implicado y la velocidad de elaboración.

La selección del SPD depende fundamentalmente del volumen previsto y características del paciente. En general, para una farmacia que desee iniciarse 
A. B. MAESTRE HERNÁNDEZ; M. GONZÁLEZ VALDIVIESO; J. ARASA; E. LÓPEZ PINTOR

ESTUDIO DE REVISIÓN DE LAS VENTAJAS E INCONVENIENTES DE LOS SISTEMAS PERSONALIZADOS

DE DOSIFICACIÓN (SPD) Y EVALUACIÓN DE SU ADECUACIÓN AL PERFIL DEL PACIENTE Y TIPO DE FARMACIA

se recomienda un SPD manual no reutilizable por la facilidad de manejo y coste mínimo. En función del nivel de elaboración y las disponibilidades de inversión, podría plantearse un SPD semiautomático. Los robots deberían reservarse para grandes volúmenes de elaboración por suponer una inversión elevada difícilmente amortizable para el actual nivel medio de elaboración de las farmacias españolas.

Palabras clave: SPD; Adherencia; Servicios Profesionales Farmacéuticos.

ABSTRACT: The Dose Administration Aids (DAA) are a useful tool to improve therapeutic adherence from the community pharmacy. However, there are many systems in the market with differentiating characteristics that you need to know in order to select the most appropriate for each pharmacy.

The objective of this work has been to conduct a review of the different types of SPD that exist, analyze the advantages and disadvantages of each and evaluate their suitability according to the desired use, patient profile and type of pharmacy. To respond to these objectives, a review of the information has been carried out by different sources and methods. Three types of SPD have been identified: manual, semi-automatic and automatic, differing fundamentally in the closure of the blister, the type of sealing, the duration, the personnel involved and the speed of preparation.

The selection of the DDA depends mainly on the expected volume and characteristics of the patient. In general, for a pharmacy that wants to start, a non-reusable manual DDA is recommended because of the ease of handling and minimum cost. Depending on the level of processing and the availability of investment, a semi-automatic DDA could be considered. Robots should be reserved for large volumes of processing because it is a high investment that is difficult to amortize for the current average level of preparation of Spanish pharmacies.

Keys words: DAA; Adherence; Community Pharmacy Services.

\section{INTRODUCCIÓN}

En la actualidad, las actividades del farmacéutico comunitario se orientan a la prestación de Servicios Profesionales Farmacéuticos Asistenciales (SPFA), definidos en España por FORO (FORO AF-FC, 2010), como «aquellas actividades sanitarias prestadas desde la Farmacia Comunitaria por un farmacéutico que emplea sus competencias profesionales para la prevención de la enfermedad y la mejora tanto de la

Ediciones Universidad de Salamanca / @@ఠ $\quad$ FarmaJournal, vol. 5, núm. 1 (2020), pp. 47-54 
A. B. MAESTRE HERNÁNDEZ; M. GONZÁLEZ VALDIVIESO; J. ARASA; E. LÓPEZ PINTOR

ESTUDIO DE REVISIÓN DE LAS VENTAJAS E INCONVENIENTES DE LOS SISTEMAS PERSONALIZADOS

DE DOSIFICACIÓN (SPD) Y EVALUACIÓN DE SU ADECUACIÓN AL PERFIL DEL PACIENTE Y TIPO DE FARMACIA

salud de la población como la de los destinatarios de los medicamentos y productos sanitarios, desempeñando un papel activo en la optimización del proceso de uso y de los resultados de los tratamientos». A nivel internacional hay varias experiencias que ponen en valor los SPF Benrimoj (2000) y Breault (2017) proponen que estos servicios sean desarrollados y explotados para el bienestar de la salud.

Los SPFA de Atención Farmacéutica se dividen en dos grupos: los orientados a mejorar el proceso de uso de los medicamentos y los orientados a mejorar los resultados de la farmacoterapia. Entre los primeros, se incluyen los orientados a la mejora de la adherencia terapéutica.

La falta de adherencia terapéutica es un problema de salud pública de magnitud mundial, cuya mejora requiere una intervención desde todos los niveles asistenciales y por todos los agentes que participan del cuidado del paciente, entre los cuales se encuentra el farmacéutico. Por ello, además de la dispensación y la indicación farmacéutica, los servicios orientados a mejorar la adherencia terapéutica probablemente sean uno de los más idóneos para ser realizados desde la Farmacia Comunitaria, y su prestación cuenta con respaldo legal. En este sentido, en España, la Ley 29/2006 de Garantías y Uso Racional de los Medicamentos y Productos Sanitarios establece en su artículo 84.1 que, en las Oficinas de Farmacia, los farmacéuticos, como responsables de la dispensación, velarán por el cumplimiento de las pautas establecidas por el médico en la prescripción y cooperarán con él en el seguimiento del tratamiento a través de los procedimientos de atención farmacéutica una vez dispensado el medicamento «[...] podrán facilitar sistemas personalizados de dosificación (SPD) a los pacientes que lo soliciten, en orden a mejorar el cumplimiento terapéutico, en los tratamientos y con las condiciones y requisitos que establezcan las administraciones sanitarias competentes».

Un Sistema Personalizados de Dosificación, SPD, es un envase especial que permite ordenar la medicación que ha de recibir un paciente durante un tiempo, teniendo en cuenta dosis y horarios, para facilitar la correcta administración de la misma. Esto evita que el paciente tenga que manipular múltiples envases, reuniendo toda la medicación que ha de tomar, debidamente organizada, en uno solo, minimizando errores de medicación y dosificación. Diferentes estudios demuestran que su utilización disminuye la morbimortalidad en los pacientes no cumplidores (Dillaa, 2009) o con problemas de prescripción inadecuada (Barris, 2015). Es, por tanto, una herramienta útil para mejorar el patrón de cumplimiento principalmente en pacientes ancianos, pluripatológicos y polimedicados, donde los olvidos o el desconocimiento constituyen la principal causa de falta de adherencia. La elaboración de SPD constituye un acto postdispensación, que debe realizarse con estricto cumplimiento de una serie de requisitos éticos y legales. El Consejo General de Colegios Oficiales de Farmacéuticos (CGCOF) publicó en el año 2018 Buenas Prácticas de Atención Farmacéutica en España en la que se encuentran unas pautas generales y un procedimiento normalizado de trabajo 
que sirven de partida a los Colegios Oficiales Farmacéuticos para llevar a cabo la elaboración de los SPD en sus correspondientes provincias (Grupo de Trabajo de Buenas Prácticas, CGCOF, 2018).

En resumen, los SPD son una herramienta útil para mejorar la adherencia terapéutica de determinados perfiles de pacientes desde la farmacia comunitaria. Sin embargo, existen en el mercado multitud de dispositivos SPD, cada uno de características diferenciadoras, así como herramientas y dispositivos que ayudan u optimizan su elaboración. La no existencia de un documento técnico que recopile toda la información sobre los tipos y características de cada uno de estos dispositivos dificulta enormemente la selección por parte de los farmacéuticos que se quieren iniciar en esta herramienta y comenzar a proveer servicios orientados a mejorar la adherencia.

El objetivo de este trabajo es realizar una revisión de los diferentes tipos de SPD que existen en el mercado farmacéutico, analizar las ventajas e inconvenientes de cada uno y valorar su adecuación en función del uso previsto, del perfil de paciente y del tipo de farmacia. Este documento servirá de ayuda a los farmacéuticos para seleccionar el SPD más adecuado a sus necesidades y contribuir a mejorar la adherencia desde la farmacia comunitaria.

\section{Metodología}

La respuesta al objetivo planteado ha requerido realizar la búsqueda y recopilación de la información utilizando diferentes fuentes y métodos.

En primer lugar, para conocer el estado actual del tema y la utilidad de los SPD en la mejora de la adherencia, se ha llevado a cabo una revisión de la literatura utilizando las siguientes bases de datos: Medline a través de PubMed, SCOPUS y Google Scholar. La búsqueda se ha completado con la consulta a expertos sobre el tema, principalmente farmacéuticos con experiencia en la elaboración de SPD y con la revisión de la bibliografía de los artículos seleccionados.

Para conocer los tipos y características de los SPD, se ha realizado una búsqueda libre en internet; ello ha permitido acceder a información técnica procedente de revistas especializadas y páginas web de fabricantes, con los que se ha contactado directamente o a través del delegado para solicitar documentación adicional y muestras de producto, por teléfono y/o por correo electrónico.

La recopilación de información y material se ha completado con la asistencia a una feria del sector farmacéutico (INFARMA, 2018), al Congreso Nacional de Farmacéuticos Comunitarios (SEFAC, 2018) y la realización de un curso de especialista en SPD impartido por la Universidad Miguel Hernández de Elche.

Finalmente, una vez elaborado el documento, fue enviado a dos farmacéuticos expertos en elaboración de SPD para evaluación y comentarios. 
A. B. MAESTRE HERNÁNDEZ; M. GONZÁLEZ VALDIVIESO; J. ARASA; E. LÓPEZ PINTOR

ESTUDIO DE REVISIÓN DE LAS VENTAJAS E INCONVENIENTES DE LOS SISTEMAS PERSONALIZADOS

DE DOSIFICACIÓN (SPD) Y EVALUACIÓN DE SU ADECUACIÓN AL PERFIL DEL PACIENTE Y TIPO DE FARMACIA

\section{Resultados y discusión}

Con este trabajo se ha realizado una revisión de los diferentes tipos de SPD con un análisis de sus ventajas e inconvenientes, determinando la adecuación de éstos a cada perfil de farmacia y de paciente, para que las farmacias comunitarias lo puedan usar de base para implantar la elaboración de SPD. Asimismo, la información contenida en este documento podría extrapolarse a otros niveles asistenciales. Este trabajo constituye una innovación de gran aplicabilidad en la práctica diaria asistencial del farmacéutico, ya que no existe, hasta nuestro conocimiento, ningún documento de estas características.

Se han identificado 3 tipos de SPD: manuales, semiautomáticos y automáticos. Dentro de cada uno de ellos existen diferentes subtipos, cuyas ventajas e inconvenientes deben tenerse en cuenta para seleccionar el más adecuado para cada farmacia y perfil de paciente.

Los SPD manuales son aquellos que deben ser elaborados íntegramente por un farmacéutico acreditado, responsable del SPD tanto a nivel asistencial como técnico. En cualquier caso, la supervisión final siempre debe ser verificada por otro farmacéutico. Presentan como ventaja que son más económicos (a modo orientativo, el coste de una caja de 100 blísteres es de 64,50€) pero, al mismo tiempo, requieren mayor tiempo y personal para la elaboración.

La Tabla 1 muestra un resumen de las características de los SPD manuales. Como puede observarse, se clasifican en SPD reutilizables y no reutilizables. Los segundos, más parecidos al concepto de "pastillero», no se recomiendan debido al riesgo de contaminación cruzada. Además, este tipo de dispositivos obligan al paciente a tener uno lleno en casa y otro vacío (dos blísteres por paciente) que llevará a la farmacia para su posterior preparación, con el consiguiente riesgo de pérdidas u olvidos.

Se han identificado hasta nueve sistemas distintos de SPD manuales no reutilizables. La diferencia fundamental entre ellos radica en el tipo de cierre (por delante o por detrás); la duración de la medicación (desde SPD de fin de semana hasta los mensuales), y en el número de alveolos por día, existiendo desde SPD para pacientes que solo tienen pautadas dos tomas al día hasta SPD que permiten hasta seis tomas diarias. Existen modelos diseñados específicamente para animales polimedicados. El sellado de estos dispositivos normalmente es en frío; en el caso de sellado en caliente se necesita una termoselladora. En algunos casos se necesita cierta destreza para abrir el dispositivo.

En general, los dispositivos manuales reutilizables son útiles para aquellas farmacias que van a iniciarse y donde se espera un pequeño volumen de elaboración.

Los SPD semiautomáticos se caracterizan por que el farmacéutico acreditado es ayudado por un máquina o sistema informático que le facilita la elaboración del SPD y ayuda a minimizar errores de elaboración. 
A. B. MAESTRE HERNÁNDEZ; M. GONZÁLEZ VALDIVIESO; J. ARASA; E. LÓPEZ PINTOR

ESTUDIO DE REVISIÓN DE LAS VENTAJAS E INCONVENIENTES DE LOS SISTEMAS PERSONALIZADOS

DE DOSIFICACIÓN (SPD) Y EVALUACIÓN DE SU ADECUACIÓN AL PERFIL DEL PACIENTE Y TIPO DE FARMACIA

La ventaja del semiautomático es que disminuyen considerablemente los tiempos de elaboración, pero sigue siendo imprescindible un farmacéutico que realice el SPD y otro que lo valide. La principal desventaja es que requieren una inversión que puede no resultar coste-efectiva. A modo de ejemplo, el coste de una máquina semiautomática es de aproximadamente $3.500 €$ y el total de la instalación, software, mantenimiento y 100 blísteres es de $4.216 €$. La Tabla 2 muestra un resumen de las características de los SPD semiautomatizados. Se han identificado hasta cuatro modelos distintos, cada uno con un software específico de gestión, y con diferencias en cuanto al cierre, sellado y duración. En algunos sistemas la forma de organizar la medicación puede ser una fuente de errores de medicación. Existe un modelo apto para personas invidentes. Se recomiendan en farmacias ya iniciadas en la elaboración de SPD, donde exista un volumen mediano y estable de elaboración, con pacientes partidarios del sistema y siempre que el farmacéutico tenga disponibilidad para la inversión.

En los SPD automatizados, un técnico acondiciona la máquina para la realización del SPD, pero la elaboración física no es llevada a cabo por un farmacéutico. Las principales ventajas son que reducen los errores de medicación, son más rápidos en el momento de actualizar el tratamiento farmacológico del paciente, requieren menor plantilla de personal y permiten la trazabilidad de la medicación emblistada. Algunos robots de SPD incluso cuentan con un sistema de seguimiento de cada dosis que permite al farmacéutico controlar las tomas que le ha hecho al paciente y garantizar que se está siguiendo bien el tratamiento. Muchos de ellos ofrecen bolsas individualizadas favoreciendo la comodidad del transporte. La desventaja principal es su elevado coste $(20.000 €$ a $200.000 €)$ y la inversión que se debe realizar en el servicio técnico de mantenimiento. Además, exigen disponer de un espacio físico para su ubicación. No existen grandes diferencias entre los distintos robots disponibles en el mercado (Tabla 2).

En general, los dispositivos automatizados son de mayor utilidad para ámbito sociosanitario u hospitalario, donde se espera un elevado volumen de elaboración, o en farmacias comunitarias con un volumen muy elevado de pacientes en tratamiento con SPD, que permita amortizar la inversión.

En resumen, la selección del SPD más adecuado para una farmacia comunitaria debe ir precedida de un proceso reflexivo de evaluación de las ventajas e inconvenientes de cada sistema y tener en cuenta el perfil de paciente al que se dirige el servicio. Para una farmacia que desee incorporar el SPD como herramienta para mejorar la adherencia de sus pacientes, se recomienda iniciarse con SPD manual no reutilizable por la facilidad de su implantación y el coste mínimo que supone. En función del nivel de elaboración y las disponibilidades de inversión, podría plantearse un SPD semiautomático. Los robots automáticos deberían reservarse para un gran volumen de pacientes puesto que suponen una inversión muy elevada difícilmente amortizable para el actual volumen medio de elaboración de la farmacia española. 
A. B. MAESTRE HERNÁNDEZ; M. GONZÁLEZ VALDIVIESO; J. ARASA; E. LÓPEZ PINTOR

ESTUDIO DE REVISIÓN DE LAS VENTAJAS E INCONVENIENTES DE LOS SISTEMAS PERSONALIZADOS

DE DOSIFICACIÓN (SPD) Y EVALUACIÓN DE SU ADECUACIÓN AL PERFIL DEL PACIENTE Y TIPO DE FARMACIA

TABLA 1. SPD semiautomático y automático: ventajas, inconvenientes y tipo

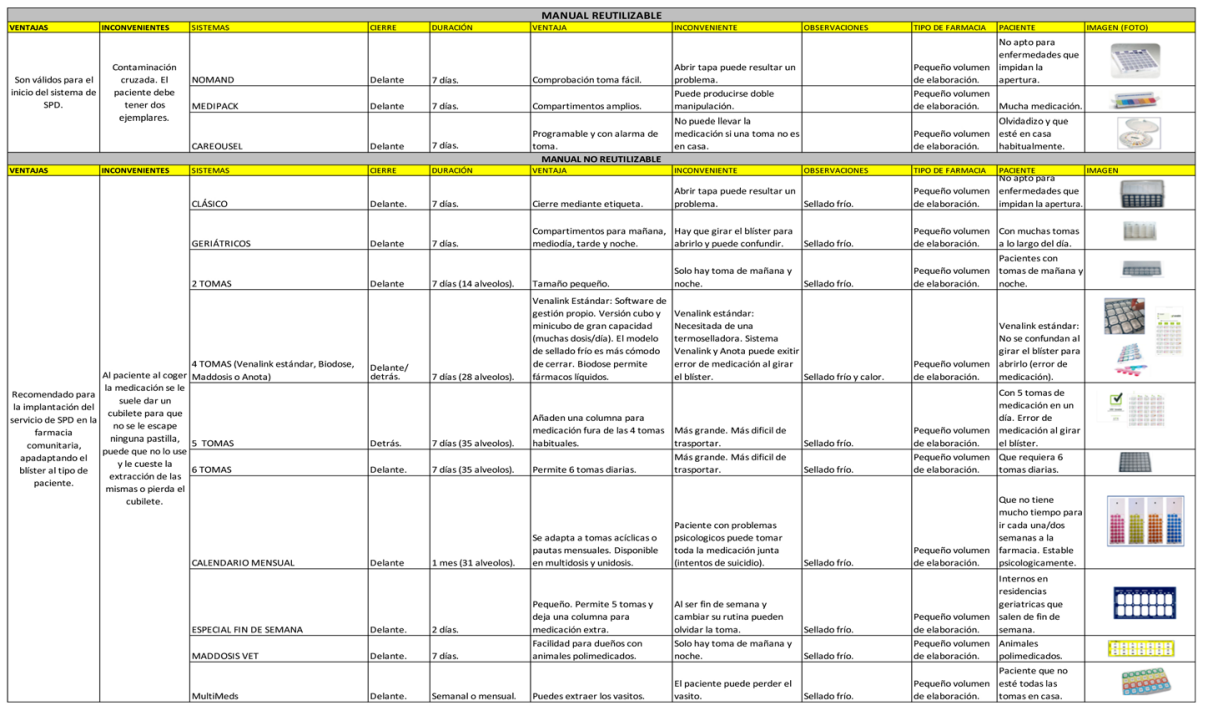

TABLA 2. SPD semiautomático y automático: ventajas, inconvenientes y tipos

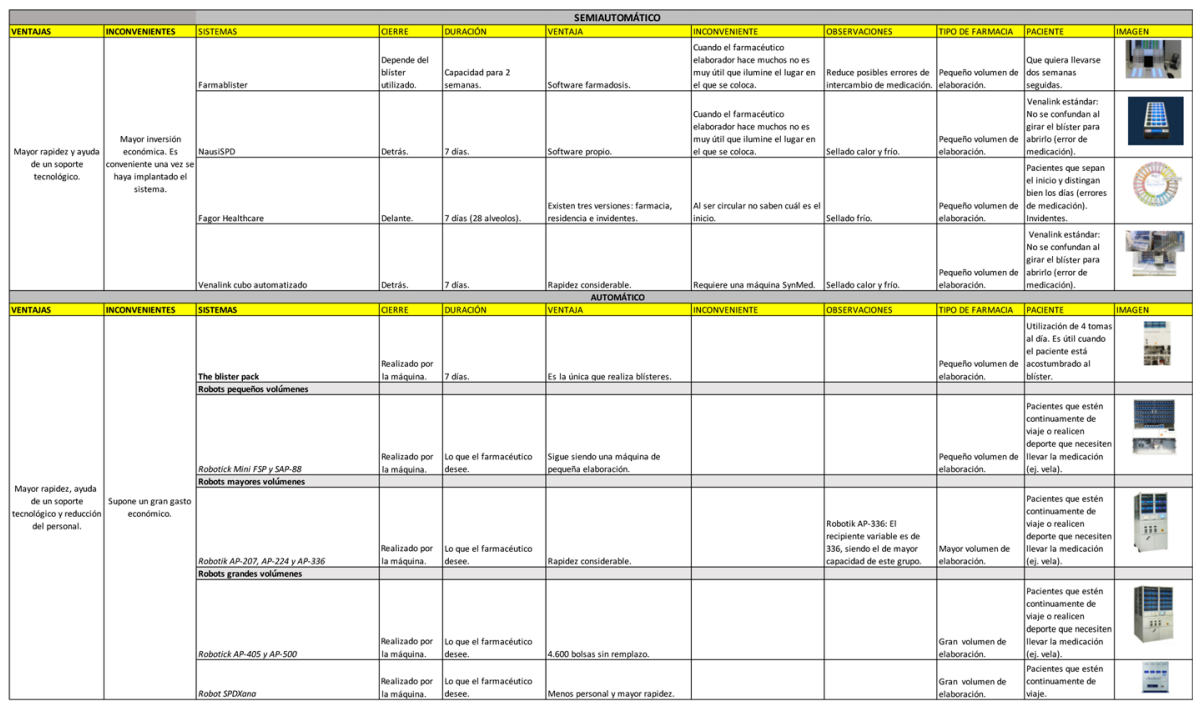

Ediciones Universidad de Salamanca / @®@

FarmaJournal, vol. 5, núm. 1 (2020), pp. 47-54 
A. B. MAESTRE HERNÁNDEZ; M. GONZÁLEZ VALDIVIESO; J. ARASA; E. LÓPEZ PINTOR

ESTUDIO DE REVISIÓN DE LAS VENTAJAS E INCONVENIENTES DE LOS SISTEMAS PERSONALIZADOS

DE DOSIFICACIÓN (SPD) Y EVALUACIÓN DE SU ADECUACIÓN AL PERFIL DEL PACIENTE Y TIPO DE FARMACIA

\section{Bibliografía}

Barris D. Revisión de medicación según los criterios STOPP/START en pacientes mayores del servicio de sistema personalizado de dosificación de medicamentos de una farmacia comunitaria. Farmacéuticos comunitarios. 2015; 7(2):31-36.

Benrimoj SI, Langford JH, Berry G, Collins D, Lauchlan R, Stewart K, et al. Economic Impact of Increased Clinical Intervention Rates in Community Pharmacy: A Randomised Trial of the Effect of Education and a Professional Allowance. PharmacoEconomics. noviembre de 2000; 18(5):459-468.

Blísters Medical Dispenser [Internet]. Fagor Healthcare. 2015 [citado 27 de febrero de 2019]. Disponible en: http://www.fagorhealthcare.com/es/blisters-medical-dispenser/.

Breault RR, Whissell JG, Hughes CA, Schindel TJ. Development and implementation of the compensation plan for pharmacy services in Alberta, Canada. Journal of the American Pharmacists Association. Julio de 2017; 57(4):532-541.

Dillaa T, Valladaresa A, Lizánb L, Sacristán JA. Adherencia y persistencia terapéutica: causas, consecuencias y estrategias de mejora. Aten Primaria. 2009; 41(6):342-348.

Foro de Atención Farmacéutica en Farmacia Comunitaria, panel de expertos. Guía práctica para los Servicios de Atención Farmacéutica en farmacia comunitaria. Mayo de 2010. Madrid: Ed. Consejo General de Colegios Oficiales de Farmacéuticos. ISBN-13: 978-84-693-1717-4.

Grupo de trabajo de Buenas Prácticas en Farmacia Comunitaria en España. Servicio de elaboración y provisión de Sistemas Personalizados de Dosificación (SPD). Madrid: Consejo General de Colegios Oficiales de Farmacéuticos; 2018.

Nausikaa eHealth and Software - NausiSPD_demo [Internet] [citado 27 de febrero de 2019]. Disponible en: http://www.nausikaaehealth.com/nausispd_demo/.

Robots SPD farmacia, sistemas personalizados de dosificación [Internet]. [citado 27 de febrero de 2019]. Disponible en: http://www.robotfarmacia.es/robots-spd-parafarmacias.html.

Rodríguez Tejonero M. ${ }^{a}$ I. Material de acondicionamiento primario de medicamentos [TESIS]. Universidad Complutense de Madrid. Facultad de Farmacia. Madrid; 2015.

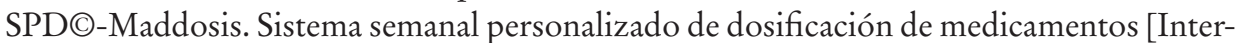
net] [citado 27 de febrero de 2019]. Disponible en: http://www.dosificacion.com/ nuestras-spds-maddosis-mnu-7.

SPD@-Venalink. Formatos del SPD Venalink | SPD Venalink [Internet]. VENALINK [citado 27 de febrero de 2019]. Disponible en: http://www.venalink.es/formatosdel-spd-venalink/. 